\title{
Fußball und Regionalentwicklung. Eine Analyse der regionalwirtschaftlichen Effekte eines Fußball-Bundesliga- Vereins - dargestellt am Beispiel des Borussia VfL 1900 Mönchengladbach
}

\author{
Rüdiger Hamm ${ }^{1}$ Angelika Jäger ${ }^{1}$ Christina Fischer ${ }^{1}$
}

Eingegangen: 17. September 2015 / Angenommen: 24. Februar 2016 / Online publiziert: 17. März 2016

(C) Springer-Verlag Berlin Heidelberg 2016

Zusammenfassung Fußball - die wichtigste Nebensache der Welt - ist nicht nur die beliebteste Sportart in Deutschland, sondern inzwischen auch Forschungsgegenstand verschiedenster Wissenschaftsdisziplinen. So finden beispielsweise die regionalwirtschaftlichen Effekte von Fußballvereinen das Interesse von Regionalökonomen und politikberatender Forschung. Denn während sich das sportliche Hauptgeschehen auf dem Platz abspielt, ergeben sich mit der Beliebtheit des Vereins steigende ökonomische Effekte für die Vereinsstandorte. Der vorliegende Beitrag analysiert auf der Basis einer Fallstudie des Erstligisten Borussia VfL 1900 Mönchengladbach, wie sich die nachfrage- und angebotsseitigen Effekte eines Fußballvereins auf seine Standortregion - in diesem Fall den Mittleren Niederrhein und die Stadt Mönchengladbach - auswirken. Einerseits werden die Wertschöpfungseffekte des Vereins Borussia VfL 1900 Mönchengladbach für die Standortregion auf fast 100 Mio. $€$ jährlich geschätzt. Zudem werden durch den Verein etwa 1.075 Arbeitsplätze gesichert. Andererseits wird im Rahmen der durchgeführten Analysen ein - grundsätzlich schwer zu quantifizierender, aber dennoch erheblicher - Effekt des Vereins auf Wahrnehmung, Bekanntheit und Image seiner Standortregion nachgewiesen. Zudem deuten die Resultate auf die Entstehung eines ,psychischen

Prof. Dr. Rüdiger Hamm

Ruediger.Hamm@hs-niederrhein.de

Angelika Jäger M.A.

Angelika.Jaeger@hs-niederrhein.de

Christina Fischer M.A.

Christina.Fischer@hs-niederrhein.de

NIERS Institut, Hochschule Niederrhein, Richard-Wagner-Straße 140, 41065 Mönchengladbach, Deutschland
Einkommens“ in der Bevölkerung hin. Zusammenfassend kann der Verein als ein bedeutender und wertvoller Standortfaktor identifiziert werden.

Schlüsselwörter Sport - Regionalentwicklung ·

Standortfaktor - Imagemessung · Multiplikatoranalyse · Stadtimage

Soccer and Regional Development. An Analysis of the Regional Economic Impacts of a Bundesliga Soccer Club-Using the Example of Borussia VfL 1900 Mönchengladbach

Abstract Soccer - the world's most important trivialityis not only the most popular sport in Europe, but has furthermore become an increasingly important research subject for various scientific disciplines. For regional economists and policy advisers, especially the estimation of regional economic impacts of soccer clubs and their regular major sport events are of high importance and interest. Whereas athletic success and challenges are achieved within the stadium, along with the popularity of the soccer club and its athletes, significant economic benefits can arise for the cities and regions hosting a soccer club. The purpose of this article is to analyze and estimate the impact, the German national league soccer club Borussia VfL 1900 Mönchengladbach exerts on its surrounding region, the Middle Lower Rhine Region in North Rhine-Westphalia, Germany. The analysis differentiates expenditure-based and supply side effects. On one hand, the value added effect for the hosting region is estimated at nearly 100 mio. $€$ annually. In addition, employment effects arise from the 1.075 employees working for the club. On the other hand, the results indicate that the club's effects on the 
Abb. 1 Regionale Effekte von Fußballvereinen. (Quelle: In Anlehnung an Hamm/Jäger/ Fischer 2014: 10)
Regionalwirtschaftliche Effekte eines Fußballvereins in der Übersicht

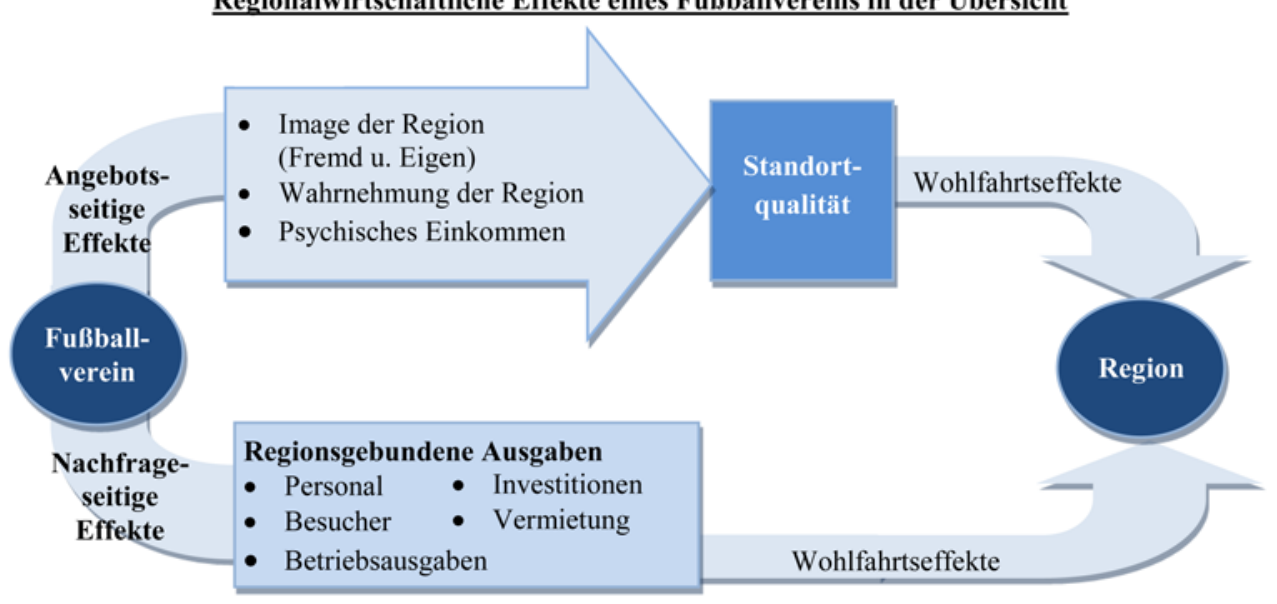

region's perception, awareness and public image - which are mostly not included in comparable scientific studies, as it is difficult to quantify and measure these effectsare significant and should no longer be neglected in costbenefit analysis of large sporting events. The results also suggest the existence of a positive influence on the local identity and sense of belonging of the population that is characterized in this study as "psychological income". Summarized, a soccer club is identified as a significant and valuable location factor.

Keywords Sports · Regional Development - Location Factor · Image Measurement · Multiplier Analysis · City Image

\section{Einleitung und Fragestellung}

Sport als „die wichtigste Nebensache der Welt“ zu bezeichnen wird heutzutage zumindest seiner ökonomischen Bedeutung kaum mehr gerecht. Im Gegenteil: Der Sport im Allgemeinen - und dies gilt ganz besonders für den professionellen Fußballsport - ist zu einem wichtigen Wirtschaftsfaktor geworden. So geht bereits im Jahr 2008 aus den Berechnungen des „Satellitenkontos Sport“ der Volkswirtschaftlichen Gesamtrechnung hervor, dass mehr als 1,7 Mio. Personen in Deutschland im Sportbereich erwerbstätig waren; dies entspricht einem Anteil von $4,4 \%$ an der Gesamtbeschäftigung. Die sportbezogene Bruttowertschöpfung erreichte damit einen ähnlich hohen Wert, wie er vom deutschen Fahrzeugbau erwirtschaftet wird (Ahlert 2013: 27, 34).

Der vorliegende Beitrag widmet sich jedoch nicht gesamtwirtschaftlichen Aspekten von Sport oder Fußball. Vielmehr geht er der Frage nach, welche Bedeutung ein renommierter Erstligaverein für die Stadt bzw. Region hat, in der er beheimatet ist, und auf welchen Wegen er der regional- wirtschaftlichen Entwicklung Impulse zu geben vermag. ${ }^{1}$ Diese Thematik wird mithilfe einer Fallstudie behandelt, in deren Mittelpunkt ein Traditionsverein des deutschen Fußballs steht - Borussia VfL 1900 Mönchengladbach. Mittlerweile wurde eine Reihe von Studien zur Analyse und Quantifizierung der regionalwirtschaftlichen Effekte von Fußballvereinen durchgeführt. Überwiegend konzentrieren sich diese Studien auf die Abschätzung der regionalen Umsatz-, Wertschöpfungs- und Beschäftigungseffekte, also auf die Bestimmung von Wirkungen, die über die Nachfrageseite ausgelöst werden (Hamm 1998; Vöpel/Steinhardt 2008; Preuß/ Könecke/Schütte 2010; Hassenpflug 2012). In den meisten Fällen gelingt diesen Arbeiten der Nachweis substanzieller nachfrageseitiger Effekte. Auch der vorliegende Beitrag behandelt und quantifiziert diese Effekte. Er geht jedoch über die Behandlung der Nachfrageseite insofern deutlich hinaus, als er außerdem Aspekte einbezieht und empirisch analysiert, die in Abbildung 1 als angebotsseitig bezeichnet werden; für sie wird in der Literatur häufig auch der Begriff der intangiblen, das heißt monetär nicht zu quantifizierenden, externen Effekte benutzt (Schwark 2004: 206). Hierzu zählen die bekanntheitssteigernde und imageverbessernde Wirkung des Fußballvereins auf die Standortkommune, die Rolle des Vereins als ein für die Standortentscheidung anderer privater Unternehmen relevanter Standortfaktor und die mögliche Entstehung eines ,psychischen Einkommens" in der Bevölkerung (Crompton 2004).

In der Regel werden angebotsseitige Effekte - wegen der herausfordernden monetären Quantifizierung - in empirischen Analysen nicht behandelt. Durch die hier gewählte Erweiterung verfolgt der vorliegende Beitrag das Ziel, ein möglichst umfassendes Gesamtbild der regional-

\footnotetext{
${ }^{1}$ Aufbau und Methodik der vorliegenden Untersuchung ähneln der Studie „Die wirtschaftliche Bedeutung des VfL Borussia Mönchengladbach für den Niederrhein“ aus dem Jahr 2006 (Hamm/JanßenTimmen/Moos 2006; unveröffentlichtes Manuskript).
} 
wirtschaftlichen Effekte eines Fußballvereins zu zeichnen. Dazu werden zunächst die nachfrageseitigen Wirkungszusammenhänge beschrieben, eine Methodik zu ihrer Abschätzung skizziert und schließlich die Ergebnisse dieser Schätzung vorgestellt (Kapitel 2). Analog wird anschließend (Kapitel 3) mit den angebotsseitigen Wirkungszusammenhängen verfahren: Nach theoretischen und methodischen Vorüberlegungen werden die Resultate der empirischen Analysen dargestellt. Eine Zusammenfassung (Kapitel 4) beschließt den Beitrag.

\section{Nachfrageseitige Effekte}

\subsection{Theoretische Überlegungen}

Ein professionell betriebener Fußballverein kann als Anbieter von Dienstleistungen gesehen werden (die folgenden Ausführungen basieren auf Crompton 1995; Hamm 1998: 43 ff.; Siegfried/Zimbalist 2000: 105 ff.; Vöpel/Steinhardt 2008: $41 \mathrm{f}$.). Zur Erstellung seines Leistungsspektrums benötigt der Verein Mitarbeiter, die Aufgaben im Bereich der geschäftlichen Abwicklung (z. B. Management), der Sportausbildung (z. B. Trainer) und der unmittelbaren sportlichen Aktivität (z. B. professionelle Fußballspieler) übernehmen. Diese Mitarbeiter beziehen Einkommen, das sie - wenn sie in der Standortregion wohnen - zum überwiegenden Teil auch dort verausgaben. Der skizzierte, von den Lohn- und Gehaltszahlungen des Sportvereins ausgehende Effekt ist jedoch nur eine der von Nachfrageimpulsen angestoßenen Wirkungsketten:

- Fußballvereine nutzen Sachkapital. Dazu gehören ein Stadion ebenso wie Trainingsanlagen oder ein Vereinsheim. Um diese Anlagen zu erstellen und zu erhalten, sind Investitionsausgaben erforderlich, die - sobald Teile der Leistungen von Unternehmen aus der Region erbracht werden - über Lohnzahlungen und Vorleistungsnachfrage zu weiteren Einkommens- und Beschäftigungseffekten innerhalb der Region führen.

- Zur Dienstleistungserstellung fragen Fußballvereine Vorleistungen nach. Der Umfang dieser Vorleistungsnachfrage dürfte - verbunden mit der Ausweitung der Aktivitätsfelder der Vereine (z. B. Verkauf von Fanartikeln, Durchführung weiterer Veranstaltungen im Stadion) - in den letzten Jahren tendenziell zugenommen haben. Soweit diese Vorleistungen aus dem regionalen Umfeld des Fußballvereins bezogen werden, werden dadurch ebenfalls regionale Einkommens- und Beschäftigungseffekte ausgelöst.

- Die Heimspiele von Fußballvereinen der ersten und zweiten Bundesliga ziehen regelmäßig Tausende von Besuchern in die Stadien. Diese Besucher kommen zum Teil aus der Region bzw. Stadt, in der die Sportveranstaltung stattfindet. In diesem Fall wird regionales Einkommen in der Region gebunden. Wenn es aber gelingt, Besucher aus entfernt gelegenen Regionen anzuziehen und wenn diese Fans am Vereinsstandort Ausgaben tätigen, dann besitzt die Vereinsaktivität Kennzeichen eines Export-Basis-Sektors: Es werden regionsexterne Einkommen in die Region gelenkt, die dort zur Sicherung von Beschäftigung beitragen.

In Folge dieser Impulse werden über die intraregionale Einkommensverausgabung und über den Bezug von notwendigen Vorleistungen aus der Region Multiplikatorprozesse angestoßen, die mit weitergehenden indirekten und induzierten Einkommens-, Vorleistungs- und Beschäftigungswirkungen verbunden sind.

\subsection{Methodik}

Die Quantifizierung der nachfrageseitigen Effekte erfolgte mithilfe einer regionalen Multiplikatoranalyse ${ }^{2}$, die mit komparativ-statischen Ansätzen die Situationen vor und nach dem Auftreten eines Impulses unter Ceteris-ParibusBedingungen vergleicht (für Einzelheiten vgl. Hamm/ Jäger/Fischer 2014: 35-61). Im konkreten Fall wurden die ökonomischen Effekte (Bruttoproduktion, Einkommen, Bruttowertschöpfung und Beschäftigung), die vom Verein Borussia Mönchengladbach auf die Stadt Mönchengladbach und die Region (Krefeld, Mönchengladbach, Kreis Viersen, Rhein-Kreis Neuss, Kreis Heinsberg) ausgehen, mit einem iterativen Multiplikatormodell geschätzt, das einige Regionsspezifika berücksichtigen kann und verfügbare Informationen über nationale Input-Output-Verflechtungen in die Rechnungen integriert. Soweit wie möglich wurde primärstatistisches Datenmaterial genutzt; wenn dies nicht möglich war, kam sekundärstatistisches Material aus amtlichen nationalen Statistiken zum Einsatz. Plausible Annahmesetzungen waren dann nicht zu vermeiden, wenn auf keinem der beiden genannten Wege die Beschaffung der notwendigen Daten möglich war.

Im Rahmen der Berechnungen wurde berücksichtigt, dass mit dem direkten Nachfrageeffekt (Beschäftigte des Vereins bzw. Wertschöpfung des Vereins) zusätzliche indirekte Nachfrageveränderungen (Sach- und Investitionsausgaben des Vereins, Ausgaben der Stadionbesucher) einhergehen und dass diese über den Einkommens- und Vorleistungskreislauf induzierte Effekte zur Folge haben. Die Stärke der Effekte wird unter anderem durch regionale Multiplikatoren beschrieben, bei denen die Summe aus direkten, indirekten und induzierten Effekten zum direkten Effekt ins Verhältnis gesetzt wird.

\footnotetext{
${ }^{2}$ Für eine detaillierte Beschreibung und kritische Auseinandersetzung mit dem Verfahren vgl. Hamm 1999: 303 ff.
} 
Direkte Effekte Je nachdem, ob man die Multiplikatoranalyse auf der Basis von Beschäftigten, Einkommen, Wertschöpfung oder Produktionswerten durchführen will, entspricht der direkte Effekt der Zahl der Beschäftigten des analysierten Fußballvereins, den von ihm gezahlten Löhnen und Gehältern, der Wertschöpfung oder dem Bruttoproduktionswert des Vereins. Im konkreten Fall hat Borussia Mönchengladbach Angaben zu der Zahl der vom Verein beschäftigten Arbeitnehmer sowie zu den Lohn- und Gehaltszahlungen zur Verfügung gestellt. In der Spielzeit 2012/13 hatte der Verein danach 211 vollzeitbeschäftigte Mitarbeiter. Hinzu kamen 920 teilzeitbeschäftigte Mitarbeiter bzw. Aushilfen, deren Zahl für die Analyse in Vollzeitäquivalente (115) umgerechnet wurde. Die gesamten Personalausgaben für diese Beschäftigten beliefen sich in der Spielzeit 2012/13 auf 44,3 Mio. €. Mithilfe von Angaben aus der Volkswirtschaftlichen Gesamtrechnung wurden diese Arbeitnehmerentgelte in einen Produktionswert umgerechnet; dieser betrug 95,8 Mio. $€$. Auf ähnlichem Weg - über den Wertschöpfungsanteil am Bruttoproduktionswert - wurde dann die Bruttowertschöpfung von Borussia Mönchengladbach auf 64,5 Mio. $€$ geschätzt.

Indirekte Effekte Die indirekten Nachfrageeffekte ergeben sich aus der regionalen Wertschöpfung und Beschäftigung, die durch die Nachfrage des Vereins nach regional erstellten Sachgütern und Dienstleistungen generiert wird. Darüber hinaus wird die durch die Nachfrage der Stadionbesucher in der Region generierte Wertschöpfung und die für ihre Herstellung erforderliche Beschäftigtenzahl hier unter den indirekten Effekten erfasst:

- Regional und sektoral differenzierte Angaben zu den Investitions- und Sachausgaben des Vereins wurden ebenfalls von der Borussia für die Spielzeit 2012/13 zur Verfügung gestellt. Der Anteil dieser Ausgaben, der am Standort Mönchengladbach bzw. in der hier analysierten Standortregion verbleibt, führt in den begünstigten Branchen der regionalen Wirtschaft in gleicher Höhe zur Bruttoproduktion. Aus den Bruttoproduktionswerten der begünstigten Branchen können - wiederum mithilfe von Angaben der nationalen Volkswirtschaftlichen Gesamtrechnung - die durch die Investitions- und Sachgüternachfrage des Vereins in der Analyseregion angestoßenen indirekten Einkommens- und Wertschöpfungseffekte ermittelt werden. Die zur Generierung dieser Wertschöpfung erforderliche Beschäftigtenzahl wurde mithilfe der nationalen Arbeitsproduktivität geschätzt. ${ }^{3}$

- Nach Angaben des Vereins haben in der Spielzeit 2012/13 mehr als 1,1 Mio. Menschen das Stadion

\footnotetext{
$\overline{{ }^{3} \text { Dies erfolgt }}$ - aufgrund der Konstruktion des verwendeten Multiplikatormodells - allerdings erst nach Berechnung aller indirekten und induzierten Effekte.
}

besucht, für deren Herkunft unterstellt wurde, dass sie der regionalen Herkunftsstruktur der Dauerkartenbesitzer entspricht. Angaben zur Höhe und Zusammensetzung der Fanausgaben außerhalb des Stadions wurden im Rahmen von Stadionbefragungen primär erhoben. Diesen Erhebungen zufolge gaben die Besucher durchschnittlich etwa $64 €$ in Zusammenhang mit einem Stadionbesuch aus - größtenteils für Anreise (35\%) und Gastronomie (31\%). Die regionale Differenzierung verdeutlicht, dass Stadionbesucher aus der Stadt Mönchengladbach nur einen deutlich niedrigeren Betrag verausgaben (etwa $22 €$ ). Nur geringfügig höher sind die Ausgaben von Stadionbesuchern, die aus der Umgebung von Mönchengladbach (Analyseregion) kommen. Darüber hinaus lassen die Erhebungen erkennen, dass die durchschnittlichen Gesamtausgaben mit wachsender Entfernung der Herkunft erkennbar zunehmen. Deshalb wurden die vom Verein bereitgestellten, nach Herkunft (Mönchengladbach, Region, „Rest der Welt") differenzierten Besucherzahlen mit den nach denselben Herkunftsregionen differenzierten durchschnittlichen Konsumausgaben multipliziert. Die so geschätzten, außerhalb des Stadions in der Stadt getätigten Gesamtausgaben von knapp 50 Mio. $€$ können jedoch kaum als zusätzliche Umsätze für die Mönchengladbacher Wirtschaft angesehen werden. Dies hängt vielmehr davon ab, wo die Stadionbesucher dieses Geld verausgabt hätten, würde es die Borussia in Mönchengladbach nicht geben. Während man davon ausgehen kann, dass die Ausgaben der Besucher, die von weit her zu einem Spiel nach Mönchengladbach kommen, ohne die Borussia kaum in Mönchengladbach getätigt worden wären, dürfte dies für die Mönchengladbacher Stadionbesucher nicht gelten. Zumindest ein Teil von ihnen hätte das Geld ebenfalls in der Stadt, jedoch für einen anderen Zweck verausgabt. Für die Modellrechnungen wurde deshalb unterstellt, dass alle Mönchengladbacher, weiter auch $60 \%$ der regionalen und $5 \%$ der überregionalen Besucher des Stadions die Ausgaben dennoch in Mönchengladbach getätigt hätten. Das (zusätzliche) Ausgabevolumen beträgt dann rund 37 Mio. $€$.

Induzierte Effekte Diese Effekte entstehen in der Region durch die regionale Verausgabung des von den direkten und indirekten Effekten herrührenden zusätzlichen Einkommens. Dabei ist aber nur der Teil von Interesse, der für konsumtive Zwecke innerhalb der Analyseregion verwendet wird, das heißt, es sind Annahmen über die Steuer-, Abgaben-, Spar- und die regionalen Importquoten erforderlich. Da gut bezahlte Spieler und Trainer eines Profi-Vereins vermutlich überdurchschnittlich hohe Abgaben-, Spar- und regionale Importquoten aufweisen, wurden im Modell neben den nationalen Durchschnittswerten vom 
Abb. 2 Ergebnisse: Direkte, indirekte und induzierte Effekte der Borussia auf die Stadt Mönchengladbach. (Quelle: Darstellung in Anlehnung an Hamm/Jäger/Fischer 2014: 127 und Vöpel/Steinhardt 2008: 41; eigene Erhebungen)

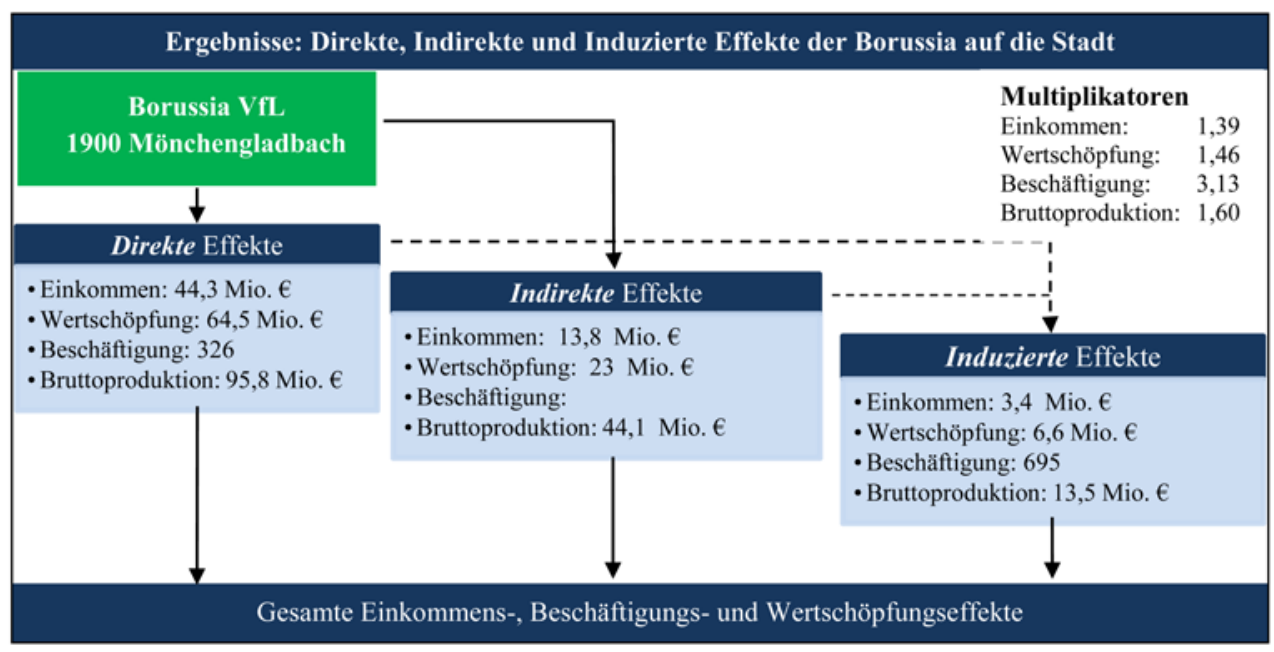

Verein bereitgestellte Informationen (regionale Verteilung der Einkommen, Abgabenquoten) und plausible Annahmesetzungen (Sparquoten, regionale Importquoten) verwendet. Die mit den Einkommen verbundene zusätzliche Konsumgüternachfrage löst weitere Produktions-, Wertschöpfungs-, Einkommens- und Beschäftigungseffekte in den analysierten Regionen aus.

Weitere induzierte Effekte entstehen schließlich „in mehreren Runden" durch die Verausgabung des Einkommens, das aufgrund der induzierten Effekte der ersten Runde entsteht. Eine andere Form induzierter Effekte wird in regionalen Multiplikatoranalysen gelegentlich vernachlässigt ${ }^{4}$ : Die Sach- und Investitionsausgaben des Vereins, aber auch alle generierten Konsumausgaben führen zum Teil in Mönchengladbach zu einem Anstieg der Bruttoproduktion, der mit einer Steigerung der Vorleistungsnachfrage verknüpft ist. Diese Vorleistungsnachfrage kann zu Aufträgen, Bruttoproduktion, Wertschöpfung und Einkommen in der regionalen Wirtschaft führen. In welchem Umfang dies geschieht, hängt zum einen davon ab, welcher Anteil der generierten Vorleistungsnachfrage durch regionale Unternehmen zufriedengestellt werden kann; sie hängt zum anderen von den regionalen Vorleistungsverflechtungen ab. Da derartige Informationen nicht verfügbar sind, wurde im hier verwendeten Multiplikatormodell versucht, diese Wirkungszusammenhänge durch Einbeziehung von Informationen der nationalen Input-Output-Rechnung (Statistisches Bundesamt 2010: 54 ff.) und plausible Annahmen über branchenspezifische ,regionale Importquoten bei der Vorleistungsnachfrage" zu berücksichtigen.

Die Abschätzung all dieser Effekte erfolgt im Multiplikatormodell durch ein iteratives Vorgehen. Die Effekte werden in jeder „Rechenrunde“ schwächer und die Iteration

\footnotetext{
${ }^{4}$ Die Berücksichtigung erhöht die Komplexität des Rechen-Modells aufgrund der Interdependenz von einkommens- und vorleistungsinduzierten Kreislaufzusammenhängen.
}

wird abgebrochen, wenn die zusätzlichen Wirkungen eine zu vernachlässigende Größenordnung (Wertschöpfungseffekte unter $10.000 €$ ) annehmen.

\subsubsection{Ergebnisse}

Abbildung 2 zeigt die Ergebnisse der Modellrechnungen für die Stadt Mönchengladbach. Danach beläuft sich die geschätzte direkte Wertschöpfung der Borussia am Standort Mönchengladbach auf 64,5 Mio. $€$. Hinzu kommen die indirekten Wertschöpfungseffekte, die durch Investitionsund Sachausgaben in Mönchengladbacher Unternehmen und Ausgaben der Fußballfans entstehen. Diese belaufen sich auf 23 Mio. $€$. Induzierte Effekte, die über Kreislaufzusammenhänge Wertschöpfung generieren, schlagen mit 6,6 Mio. $€$ zu Buche. Insgesamt belaufen sich die Wertschöpfungseffekte somit auf 94,1 Mio. €. Der Wert des Wertschöpfungsmultiplikators beträgt 1,46, das heißt, jeder Euro Wertschöpfung, den der Verein unmittelbar generiert, führt in der Stadt zu einer weiteren Wertschöpfung von fast 50 Cent. Zudem entstehen - neben den direkten Einkommenszahlungen von 44,3 Mio. $€$ - durch den Verein zusätzliche Einkommen in Höhe von 17,2 Mio. $€$. Demnach beträgt der Einkommensmultiplikator 1,39. Zur Entstehung dieser Wertschöpfung werden Arbeitskräfte benötigt. Von den insgesamt rund 1.000 in der Stadt gesicherten Arbeitsplätzen verfügen einige Mitarbeiter des Vereins über ein deutlich überdurchschnittliches Einkommen. Deutlich näher am Durchschnittseinkommen entlohnt werden die über indirekte und induzierte Wirkungen gesicherten Arbeitsplätze in den Bereichen Handel, Verkehr, Nachrichten und Gaststätten.

In der Region Mittlerer Niederrhein ${ }^{5}$ hat die geschätzte direkte Wertschöpfung dieselbe Höhe wie in der Stadt

\footnotetext{
${ }^{5}$ Die Wirtschaftsregion Mittlerer Niederrhein umfasst die Städte Krefeld und Mönchengladbach sowie den Kreis Viersen, den Kreis Heinsberg und den Rhein-Kreis Neuss.
} 
Mönchengladbach: 64,5 Mio. $€$. Die indirekten Wertschöpfungseffekte betragen hier 24 Mio. $€$ und die induzierten 9 Mio. $€$. Insgesamt ergeben die Wertschöpfungseffekte am Mittleren Niederrhein einen Wert von 97,8 Mio. $€$. Der Wertschöpfungsmultiplikator nimmt damit einen Wert von 1,52 an. Neben den direkten Einkommenszahlungen von 44,3 Mio. $€$ entstehen in der Region noch weitere Einkommen in Höhe von 18,8 Mio. €. Demnach beträgt der Einkommensmultiplikator 1,42. Insgesamt wären 1.075 Arbeitskräfte für die Entstehung dieser Wertschöpfung erforderlich.

\section{Angebotsseitige Effekte}

\subsection{Theoretische Überlegungen}

Fußballvereine können eine Stadt bzw. Region überregional bekannt machen (Siegfried/Zimbalist 2000: 109). Durch die Berichterstattung über Ligaspiele ist auch die Stadt bzw. Region regelmäßig in den regionalen, nationalen, teilweise sogar in den internationalen Medien präsent. Folge hiervon dürfte ein national wie international steigender Bekanntheitsgrad der Stadt sein. Die möglichen weitergehenden Wirkungen werden in der Fachliteratur allerdings eher kritisch hinterfragt:

- Der gestiegene Bekanntheitsgrad könnte das Interesse Auswärtiger an einem Besuch in der Stadt erhöhen. Ein steigendes Tourismusaufkommen wäre mit Umsatzsteigerungen bei regionalen Unternehmen verbunden, insbesondere in den Bereichen Handel und Gastronomie.

- Der gestiegene Bekanntheitsgrad kann auch zur Folge haben, dass das Interesse von Unternehmen für den Wirtschaftsstandort geweckt wird und dieses gestiegene Interesse letztlich $\mathrm{zu}$ Unternehmensansiedlungen führt (Crompton 2004: 43 f.). Dies wäre mit einer höheren regionalen Wertschöpfung und besseren Beschäftigungsmöglichkeiten verbunden.

Ein weiterer möglicher Effekt ist eine imageverbessernde Wirkung. Das Image ist „,...) die Gesamtheit von Gefühlen, Einstellungen, Erfahrungen und Meinungen bewusster und unbewusster Art, die sich eine Person bzw. eine Personengruppe von einem ,Meinungsgegenstand' (z. B. einem Produkt, einer Marke, einem Unternehmen) macht" (Essig/ Soulas de Russel/Semanakova 2003: 159). Im vorliegenden Fall ist der Meinungsgegenstand eine Region bzw. eine Stadt.

Das regionale Image wird in regionalökonomischen Erklärungsansätzen (Diller 1991: 29 f.; Grabow 1994: 148 ff.) als eigenständiger ,weicher“, die Unternehmensentscheidungen mit bestimmender Standortfaktor eingeschätzt. Auf Unternehmensbefragungen basierende Untersuchungen bestätigen, dass das regionale Image von Unternehmen als ein wichtiger Standortfaktor angesehen wird (Hamm/Vetter 2004; Hamm/Wenke/Növer et al. 2013: 17). Sportvereine und -einrichtungen spielen in diesem Zusammenhang eine wichtige Rolle (Crompton 2004: 44 f.):

- Haben früher oftmals die industriellen Schwerpunkte einer Region deren Image geprägt, so wurden es mit dem Wandel zur Dienstleistungsgesellschaft die Wolkenkratzer der Dienstleistungsunternehmen. Bei einem fortschreitenden Wandel hin zu freizeitorientierten Diensten könnten Sportvereine bzw. Sporteinrichtungen zu den „Image Builders“ der Zukunft werden.

- Die Relevanz, die ein Sportverein bzw. eine Sporteinrichtung für das Image einer Stadt haben wird, dürfte darüber hinaus von der Größe und ,sonstigen Wichtigkeit“ der Kommune oder Region abhängen. Die Imageeffekte eines Fußballvereins werden für eine Stadt wie Kaiserslautern, Bielefeld oder Mönchengladbach von größerer Relevanz sein als für München, Hamburg oder Berlin. Denn in den zuletzt genannten Städten ist der Fußball-Bundesligist Teil eines umfangreichen, zumeist überregional bekannten „Kultur- und FreizeitAngebots“, während er in den anderen Fällen im Mittelpunkt eines solchen Angebots steht.

- Im Fall von Ligasport wird die Positionierung von Sportvereinen als symptomatisch für die Positionierung der Stadt angesehen: Spielt z. B. Borussia Mönchengladbach in der ersten Bundesliga, so spielt auch die Stadt in der obersten Liga der deutschen Städte; würde die Borussia in die zweite Liga absteigen, so würden sich auch die Stadt und viele ihrer Einwohner als Verlierer und von nun an zweitklassig fühlen.

Durch Steigerungen des Bekanntheitsgrades und Verbesserungen des regionalen Images können möglicherweise mehr Besucher, mehr Bewohner und mehr wirtschaftliche Aktivität in die Region gelenkt bzw. generiert werden. Das wiederum könnte dazu führen, dass Fußballvereine für die Standortentscheidung anderer privater Unternehmen $\mathrm{zu}$ einem relevanten Standortfaktor werden. Sportvereine bzw. Sporteinrichtungen sind aber auch ein unmittelbares Element der regionalen Standortbedingungen und können so über die Attrahierung zusätzlicher Unternehmensansiedlungen die regionalwirtschaftliche Entwicklung stimulieren (Hamm 1998: 45 f.; Dietl/Pauli 1999: 27 ff.; Crompton 2004: 46 ff.). Hierbei können zwei unterschiedliche Wirkungszusammenhänge Relevanz haben (Hamm 1998: 45; Crompton 2004: 48)6:

\footnotetext{
${ }^{6}$ Crompton (2004) unterscheidet drei Arten, durch die die regionalwirtschaftliche Entwicklung stimuliert werden kann: „Proximate development" und „complementary development" nach Crompton sind den direkten Sogwirkungen vergleichbar, ein ,general development", wie Crompton es beschreibt, ergibt sich über indirekte Sogwirkungen.
} 
- Direkte Sogeffekte: Sie ergeben sich dann, wenn der impulsgebende Bereich, also der Fußballverein, unmittelbare Sogeffekte auf andere Unternehmen ausübt. Beispiele direkter Sogwirkungen von Sportvereinen sind der Verkauf bzw. Versand von Fanartikeln, Gastronomiebetriebe im Bereich der Sporteinrichtungen sowie regionale Reiseveranstalter, die die Fanreisen zu den Auswärtsspielen organisieren. Da Fußballbundesligaspiele große Besucherzahlen anziehen, wäre es über diese Aktivität hinaus denkbar, dass andere Sport- und Freizeiteinrichtungen, der Handel mit Sportbekleidung und -geräten oder Betriebe des Hotel- und Gaststättengewerbes die räumliche Nähe zu dem zugehörigen Stadion suchen. In diesem Fall könnte man das Stadion als impulsgebenden Kern bezeichnen, durch dessen Sogkraft andere Unternehmen und Einrichtungen angezogen werden.

- Indirekte, angebotsseitige Impulse: Sie können auftreten, weil das kommunale bzw. regionale Sport- und Freizeitangebot zu den weichen Standortfaktoren zählt. Die damit einhergehende Verbesserung der Ausstattung mit weichen Standortfaktoren steigert die Attraktivität der Region. $\mathrm{Zu}$ fragen ist, ob die Existenz eines attraktiven Fußballbundesligisten mit den entsprechenden Sportstätten die Standortentscheidungen anderer Unternehmen beeinflusst. Einige Überlegungen lassen dies eher fraglich erscheinen: Zum einen ist die Zahl der Faktoren, die die Standortentscheidungen von Unternehmen und die betrieblichen Entwicklungen beeinflussen, recht hoch; das Sport- und Freizeitangebot ist hiervon nur ein Aspekt und ein Fußballverein ist in diesem Sport- und Freizeitangebot wiederum nur ein Teilelement. Zum anderen kommen befragungsbasierte Analysen zur Bedeutung einzelner Standortfaktoren zu dem Resultat, dass weiche Standortfaktoren insgesamt nur eine nachrangige Relevanz besitzen (Grabow 1994: 147 ff.; Hamm/Wenke/Növer et al. 2013).

Neben den vorgenannten Wirkungen, die sich hauptsächlich auf regionsexterne Zielgruppen beziehen, nimmt der Fußballverein aber auch in Form des „Psychischen Einkommens“" (Crompton 2004: 49) Einfluss auf die Regionsbewohner. Der Fan eines Fußballvereins wird Freude an dem Verein haben und kann sich mit ihm als „seinem“ Verein identifizieren, sogar ohne jemals selbst einem Spiel beizuwohnen. Fußballinteressierte informieren sich in den Medien über Spielergebnisse und vereinsrelevante Neuigkeiten, um in der Folge mit Familie, Freunden oder Arbeitskollegen über „,ihr Team“ zu diskutieren (Crompton 2004: 49). Fußballvereine können Identifikationsobjekte darstellen, die je nach Stadt und Region im Laufe der Zeit eine mehr oder weniger wichtige Bedeutung für die Begründung und Entwicklung der regionalen Identität eingenommen haben. Profifußballteams und ihre Stadien können als „Kristallisationspunkte eines erwachenden und wachsenden regionalen Gemeinschaftsbewusstseins“ (Luh 2002: 203) betrachtet werden. Insbesondere für Städte mit einem Mangel an geschichtlicher Tradition und städtebaulichen Wahrzeichen stellen Bundesligavereine wichtige regionale Identifikationsobjekte dar. Sie sind ein regionsspezifisches Symbol, auf das die Einwohner stolz sein können (Schmidt/ Bünning 2012: 14).

\subsection{Methodik}

Um die in Abschnitt 3.1 beschriebenen angebotsseitigen Wirkungsketten empirisch zu überprüfen, wurde im Wesentlichen das Instrument empirischer Befragungen eingesetzt. Die dadurch gewonnenen - eher qualitativen Erkenntnisse können eine Beurteilung der nicht monetär quantifizierbaren Effekte zumindest erleichtern. Für die vorliegende Untersuchung wurden Stadion-, Passanten- und Onlinebefragungen durchgeführt. Es wurde eine Stichprobe von insgesamt 1.757 Befragungsteilnehmern generiert, die valide Aussagen zulässt:

- Stadionbesucher: Während dreier Heimspiele von Borussia Mönchengladbach in der Bundesligasaison 2013/2014 wurden insgesamt 913 Heim- und Auswärtsfans befragt. ${ }^{7}$ Durch die Stadionbefragungen sollten die Wahrnehmungs- und Imageeffekte des Vereins bei stark fußballaffinen Personen untersucht werden.

- Personen aus dem direkten Einzugs- und Einflussgebiet des Vereins: Die hierzu durchgeführten Passantenbefragungen (z. B. in Viersen, Krefeld, Heinsberg und Mönchengladbach) sollten dazu dienen, Bekanntheit, Wahrnehmungs- und Imageeffekte sowie das ,psychische Einkommen" bei der regionalen Bevölkerung zu untersuchen. Es wurden im Oktober und November 2013579 Probanden mittels einer papierbasierten, persönlichen Passantenumfrage in den Innenstädten der genannten Kommunen befragt.

- Onlinebefragung: Mit der bundesweit angelegten Onlinebefragung sollte ein Gegengewicht zu den befragten Fans und Passanten in der Fanregion geschaffen werden, damit neben dem Eigenimage und der Wahrnehmung des Vereins in seinem unmittelbaren Einzugsgebiet auch der Effekt bei Personen aus entfernteren Regionen untersucht werden konnte. Über die Onlinebefragung wurden im Zeitraum von Oktober bis November 2013 insgesamt 265 Befragungsteilnehmer generiert.

\footnotetext{
${ }^{7}$ Die papierbasierte Face-to-Face-Stadionbefragung wurde am 05.10.13 $(n=410)$ im Bundesligaspiel gegen Borussia Dortmund, am 27.10.13 $(n=223)$ beim Heimspiel gegen Eintracht Frankfurt und am 09.11.13 $(n=280)$ gegen den 1. FC Nürnberg durchgeführt. Zehn Studierende führten die Befragungen unter Anleitung eines Projektverantwortlichen durch.
} 
Abb. 3 Einteilung der Befragten in Cluster. (Quelle: Eigene Darstellung auf Basis der durchgeführten Erhebungen, $n=1.198$ )

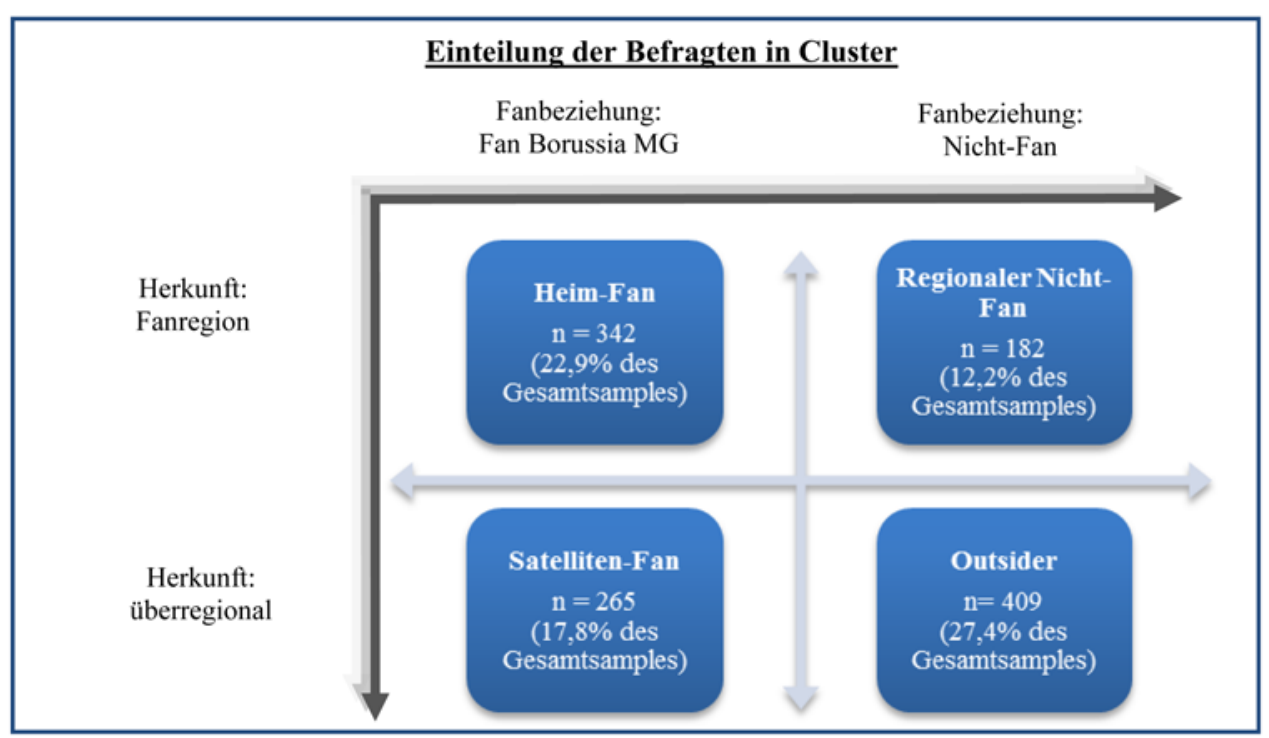

Die Wahl der Stichprobenmethode und des Befragungsvorgehens wurde darauf ausgerichtet, für die Messung der nachfrage- (hier waren Stadionbefragungen vonnöten) und der angebotsseitigen Effekte eine angemessen große und heterogene Stichprobe zu erfassen. Eine weiterführende Selektion der potenziellen Probanden z. B. im Sinne eines mehrstufigen oder geschichteten Auswahlverfahrens wurde nicht vorgenommen, die Onlinebefragung entsprach dem Vorgehen des „Convenience Sampling“ (Misoch 2015: 193), ${ }^{8}$ bei der Passantenbefragung wurde eine willkürliche Auswahl der Passanten vorgenommen, die die Innenstädte frequentierten. Bei der Stadionbefragung wurde die willkürliche Auswahl um das Selektionskriterium der Anwesenheit im Stadion erweitert. Es ist nicht auszuschließen, dass die verwendete Methodik zu einer nicht repräsentativen Stichprobe führte - Verzerrungen könnten z. B. durch das „Convenience Sampling“ bestehen. Diesem Umstand wurde durch eine geschichtete Analyse begegnet, bei der das Sample getrennt nach ergebnisbeeinflussenden Selektionscharakteristika ${ }^{9}$ untersucht wurde. Für die Analyse der angebotsseitigen Effekte wurde daher nicht in allen Fällen das Gesamtsample ausgewertet. Häufig wurde mit einem verkleinerten Sample gearbeitet, das nur die Befragungsteilnehmer der Passanten- und Onlinebefragung umfasste $(n=844)$. Dieses Vorgehen wurde gewählt, weil die Befürchtung bestand, dass die Stadionbefragung bezüglich einiger angebotsseitiger Effekte verzerrte Ergebnisse liefern könnte. Da der Anteil der Borussen-Fans unter den Befragten im Stadion deutlich überrepräsentiert war, blieben

${ }^{8}$ Eine doppelte Befragung von Probanden wurde ausgeschlossen.

${ }^{9}$ Dies sind im vorliegenden Fall primär die geographische Herkunft und der Fanstatus, siehe Erläuterungen im weiteren Text. die Befragten der Stadionbefragung im verkleinerten Sample unberücksichtigt.

Eine andere Modifikation betraf die Zuordnung der Befragten zu vier Clustern, die als „Heim-Fans“, „,regionale Nicht-Fans“, „Satelliten-Fans" und als "Outsider" bezeichnet wurden (s. Abbildung 3). Diese Einteilung verfolgte das spezielle Analyseziel herauszuarbeiten, welchen Einfluss die zwei zentralen Einflussdeterminanten „Intensität der Fanbeziehung“ und „Herkunft der Befragten“ auf die Ergebnisse haben. Die Fanbeziehung ist diesbezüglich von Interesse, da davon auszugehen ist, dass Fans die Effekte des Vereins positiver und intensiver wahrnehmen als Nicht-Fans. Die Herkunft der Befragten ist von Bedeutung hinsichtlich der Imageanalyse, um das Eigen- und Fremdimage der Stadt zu unterscheiden (siehe Abbildung 3). Als direkte Fanregion wurden hier die Stadt Mönchengladbach sowie die umliegenden Kreise Viersen, Neuss und Heinsberg betrachtet. ${ }^{10}$ Die Clusterung umfasste nur Befragte, die sowohl Angaben zu ihrem Wohnsitz als auch zu ihrer Fanbeziehung gemacht haben. Zudem wurden Befragte nicht betrachtet, die sich (nur) als „Anhänger bzw. Sympathisanten" des VfL einschätzten, da sie weder den Fans noch den Nicht-Fans klar zugeordnet werden konnten. Die Clusterung umfasste 1.198 Befragte.

\subsection{Ergebnisse}

Die Ergebnisse der Befragungen zeigen, dass die Befragten die angebotsseitigen Wirkungen, die vom Verein Borussia VfL 1900 Mönchengladbach ausgehen, stärker einschätzten als die monetären Effekte. Abbildung 4 zeigt die Bewertungen

\footnotetext{
${ }^{10}$ Diese Abgrenzung wurde anhand der regionalen Verteilung der Dauerkartenbesitzer der Borussia vorgenommen.
} 
Abb. 4 Bewertung der angebotsseitigen Effekte von Borussia Mönchengladbach. (Quelle: Eigene Darstellung auf der Basis der durchgeführten Erhebungen, $n=844$ (Passantenund Onlinebefragung))

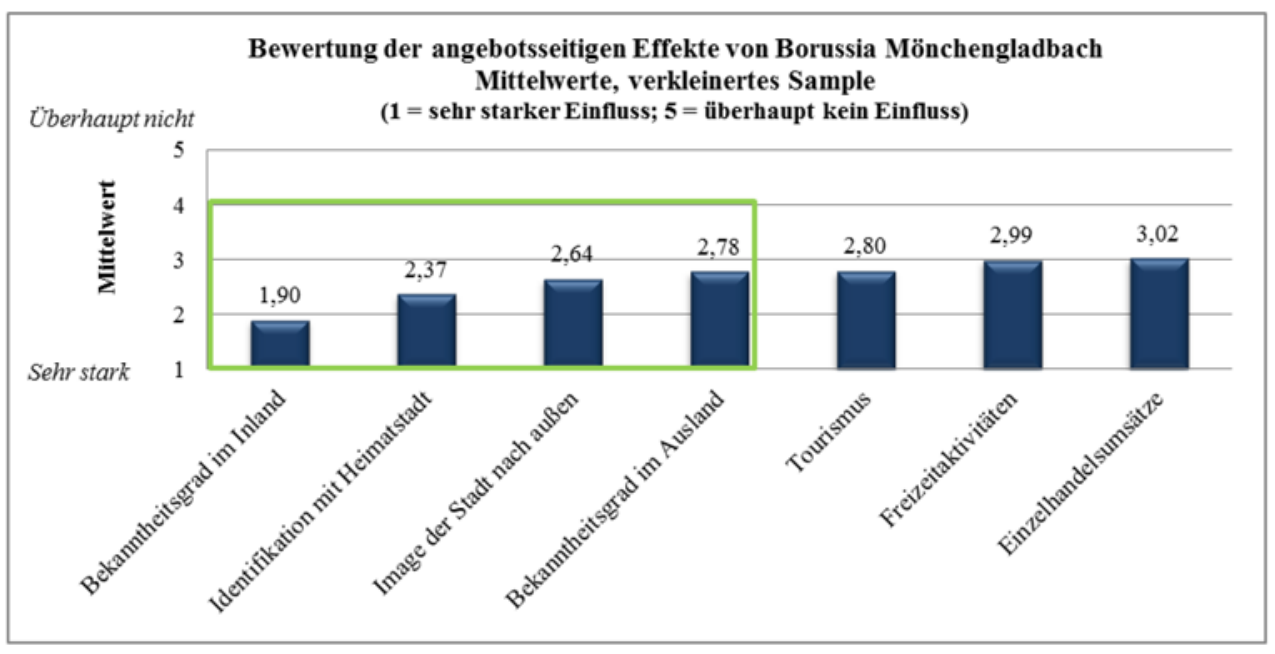

der verschiedenen Effekte durch die Befragten. Der am stärksten eingeschätzte Effekt (der niedrigste Betragswert) stellt die Bekanntheitsgradsteigerung im Inland dar, gefolgt von der Identifikation mit der Heimatstadt, dem Image der Stadt nach außen und der Bekanntheitssteigerung im Ausland. Am schwächsten wurde der Effekt auf die Einzelhandelsumsätze eingeschätzt.

\subsubsection{Bekanntheitssteigerung im In- und Ausland}

Zunächst wurde geprüft, wie der Effekt des Vereins auf die Wahrnehmung und Bekanntheit der Stadt Mönchengladbach im In- und Ausland einzuschätzen ist. In Abbildung 5 sind die Antworten der Befragten auf die Frage ausgewertet, ob die Existenz des Vereins Borussia Mönchengladbach den Bekanntheitsgrad der Stadt im Inland fördert. Abbildung 6 stellt die Ergebnisse im Hinblick auf den Bekanntheitsgrad im Ausland dar.

Insgesamt $80,3 \%$ der Befragten des verkleinerten Samples waren der Ansicht, dass der Verein den Bekanntheitsgrad der Stadt im Inland erheblich fördert. Einen starken bis sehr starken bekanntheitsfördernden Effekt im
Ausland sahen dagegen lediglich $41 \%$ des verkleinerten Samples, was vermutlich auch darauf zurückzuführen sein dürfte, dass die Borussia in den Saisons vor der Befragung nur unregelmäßig an internationalen Wettbewerben teilgenommen hatte. Bezüglich beider Effekte gilt: Je intensiver die Fanbeziehung der Befragten ist, desto höher wurden die bekanntheitssteigernden Effekte des Vereins für die Stadt eingeschätzt. Die Gruppen der Heim- und Satellitenfans stuften diesen Effekt demnach deutlich stärker ein als die Gruppen der regionalen Nicht-Fans und der Outsider. Bei der Untersuchung der bekanntheitsfördernden Effekte des Vereins im Ausland ist der Unterschied in der Bewertung durch Fans und Nicht-Fans wesentlich deutlicher zu erkennen als bei der Betrachtung der Effekte im Inland.

\subsubsection{Image}

Ein städtebauliches Merkmal oder eine zentrale regionale Institution kann die Eigenschaften, die die Öffentlichkeit damit verbindet, auf die Region übertragen. Im Rahmen der Befragung sollten die Befragten angeben, welche in Mönchengladbach bekannten Gebäude bzw. Einrichtungen
Abb. 5 Effekt des VfL Borussia Mönchengladbach auf den Bekanntheitsgrad der Stadt im Inland. (Quelle: Eigene Darstellung auf der Basis der durchgeführten Erhebungen)

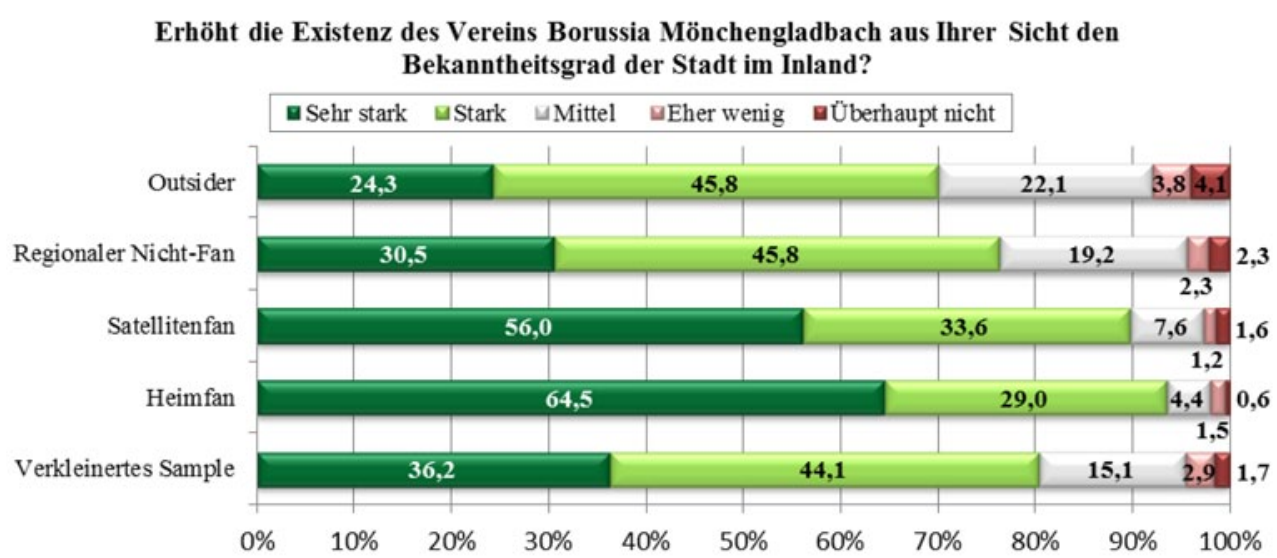


Abb. 6 Effekt des VfL Borussia Mönchengladbach auf den Bekanntheitsgrad der Stadt im Ausland. (Quelle: Eigene Darstellung auf der Basis der durchgeführten Erhebungen)

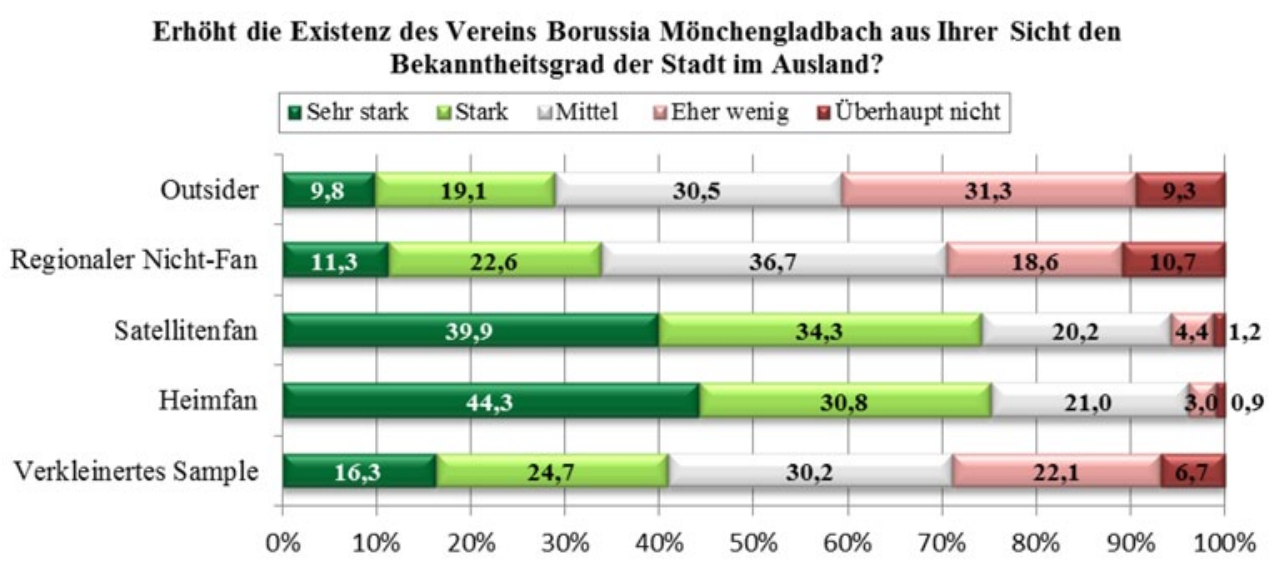

sie als regionale Imageträger bzw. Wahrzeichen bezeichnen würden. Die Ergebnisse zeigen, dass der Verein Borussia Mönchengladbach als wichtigstes Wahrzeichen der Stadt angesehen wird - bei allen befragten Gruppen erreicht der Verein mit Abstand den höchsten Grad an Zustimmung. Am größten war die Zustimmung erwartungsgemäß bei den beiden Fan-Gruppen, hier war auch der Abstand zum nächstwichtigen Imageträger sehr viel deutlicher ausgeprägt. Die Heimfans bzw. regionalen Nicht-Fans schätzten alle in die Befragung einbezogenen Institutionen (zur Auswahl standen u. a. Schloss Rheydt, Warsteiner Hockeypark/ SparkassenPark, Hochschule Niederrhein) eher als einen Imageträger ein als ihr jeweiliges überregionales Pendant. Grund hierfür könnte der bessere Kenntnisstand über die abgefragten regionalen Wahrzeichen sein. Es fällt aber auf, dass die nicht in der Region beheimateten Satellitenfans auch eher bereit waren, alle anderen Wahrzeichen als regionale Imageträger einzuschätzen als die Outsider. Dies deutet darauf hin, dass die Fanbeziehung dazu führt, dass auch Personen von außerhalb sich eher mit der Stadt und ihren Wahrzeichen beschäftigen.

Der Verein Borussia Mönchengladbach wurde in der Befragung als wichtigster Imageträger der Stadt Mönchengladbach identifiziert. Es stellte sich daher die Frage, ob der Verein zu einer Imageverbesserung der Stadt führen kann. $76,5 \%$ der Heimfans und 80,7\% der Satellitenfans sahen einen starken bis sehr starken imageverbessernden Effekt. Die regionalen Nicht-Fans und Outsider waren diesbezüglich verhaltener. Von den regionalen Nicht-Fans waren $36,6 \%$ und von den Outsidern $38,4 \%$ von einer starken bis sehr starken Imageverbesserung für die Stadt durch die Borussia überzeugt. Auffällig ist, dass der Imageeinfluss von den regionsexternen Befragten höher eingeschätzt wurde als durch die heimischen Befragten. So stimmten die Outsider der Imageverbesserung eher $\mathrm{zu}$ als die regionalen NichtFans, die Satellitenfans hatten eine leicht positivere Sicht als die Heimfans. Eine mögliche Erklärung mag darin bestehen, dass der Effekt des Vereins auf das Fremdimage (Image der Stadt nach außen) von den Einwohnern Mönchengladbachs nicht so gut eingeschätzt werden konnte. Die Auswärtigen, deren Bild der Stadt das Fremdimage ausmacht, nahmen diesen Effekt vermutlich eher wahr und konnten ihn besser beurteilen.

Ähnlich wie Unternehmen können auch Fußballvereine bestimmte Imageausprägungen und eine Markenidentität besitzen. Besonders im Profifußball ist diese oftmals geprägt von der Vereinsgeschichte und -tradition: Neben den aktuellen sind auch sportliche Erfolge der Vergangenheit (Borussia Mönchengladbach, FC Bayern München), traditionelle Konzernzugehörigkeiten (Bayer 04 Leverkusen, VfL Wolfsburg) und die Verwurzelung in bestimmten Gesellschaftsschichten („Arbeiterclub“ FC Schalke 04) besonders einprägsame Attribute, die das Image der Vereine nachhaltig beeinflussen. Auf dieser Basis kann davon ausgegangen werden, dass das Image von Borussia Mönchengladbach einerseits von den sportlichen Erfolgen der Vergangenheit und dem damaligen Image als „Fohlenelf“ (erfolgreich, begeisternd, jung, dynamisch) geprägt sein wird. Auch Attribute wie „sympathisch“, „bodenständig“ und „weltoffen“ mögen auf den Verein zutreffen (vgl. Abbildung 7 - kleinere Betragswerte bedeuten einen höheren Zustimmungsgrad). Zudem zeichnet eine Studie der ServiceValue GmbH in Zusammenarbeit mit der „Welt am Sonntag“ und der Goethe-Universität Frankfurt die Borussia als familienfreundlichsten Fußballverein aus (ServiceValue GmbH 2013).

Diese Überlegungen gaben den Ausschlag für die Wahl der in den Befragungen benutzten Attribute, deren durchschnittliche Bewertungen für Stadt und Verein in Abbildung 7 dargestellt sind. Zunächst wird klar, dass die Befragtengruppe den Verein bezüglich aller abgefragten Attribute deutlich positiver als die Stadt beurteilte. Nur das einzige negative Attribut in der Befragung (,langweilig“) wurde von den Befragten stärker mit der Stadt als mit dem Verein in Verbindung gebracht. Sowohl die Stadt als auch die Borussia wurden in Bezug auf die Eigenschaft „sympathisch“ am positivsten bewertet. Die zweite Position bei der Borussia belegte ,jung, dynamisch“, was unter anderem auf die 
Abb. 7 Eigenschaften von Stadt und Verein im Vergleich - verkleinertes Sample. (Quelle: Eigene Darstellung auf der Basis der durchgeführten Erhebungen, $n=844$ (Passantenund Onlinebefragung))
Eigenschaften von Stadt und Verein im Vergleich Mittelwerte, verkleinertes Sample

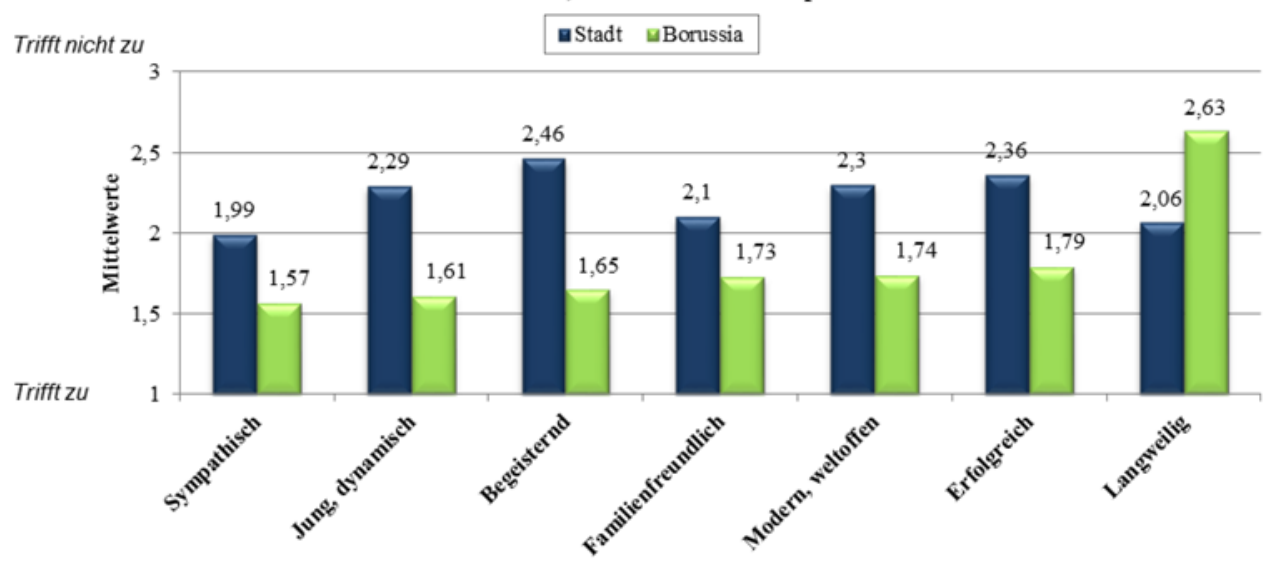

Nachwuchsspieler zurückzuführen sein dürfte, die der Verein in den letzten Jahren hervorgebracht hat. Bezüglich der Familienfreundlichkeit beurteilten die Befragten den VfL mit 1,73. Vor dem Hintergrund, dass Borussia Mönchengladbach in der Studie „Familienfreundliche Unternehmen 2013“ als familienfreundlichster Fußballverein ausgezeichnet wurde, ist dieses Befragungsergebnis eher als zurückhaltend zu bezeichnen. Die Stadt Mönchengladbach auf der anderen Seite wurde von den Befragten am ehesten als sympathisch, nachfolgend als langweilig, familienfreundlich und jung/dynamisch eingeschätzt.

Nach der Betrachtung der Eigenschaften von Verein und Stadt wird nun der Frage nachgegangen, ob die Daten einen Imagetransfer vom Verein auf die Stadt erkennen lassen. Hierzu werden die Eigenschaften von Stadt und Verein im Vergleich und nach ausgewählten Fanclustern (Einschätzungen der Satellitenfans und der Outsider) betrachtet. Genutzt wird dazu eine in Abbildung 8 ersichtliche PortfolioDarstellung, auf der die durchschnittlichen Bewertungen der betrachteten Attribute eingetragen wurden. Der Wert auf der y-Achse zeigt, wie die Befragten den Verein bewerteten, auf der $\mathrm{x}$-Achse ist die Bewertung der Stadt eingetragen.

Sollte tatsächlich ein Imagetransfer der positiven Einschätzung des Vereins auf die Stadt Mönchengladbach stattfinden, ist zu erwarten, dass die Fans der Borussia nicht nur den Verein, sondern auch die Stadt deutlich positiver bewerten als die Nicht-Fans. Diese Erwartung müsste insbesondere auf die Befragten zutreffen, die außerhalb der Fanregion wohnhaft sind. Da sie im Allgemeinen nur einen geringen Informationsstand über die Stadt Mönchengladbach besitzen, müsste der Verein für sie das zentrale Medium für den Informationstransfer und zur Imagebildung sein. Beurteilen also die regionsexternen Satellitenfans die Stadt deutlich besser als die Gruppe der Outsider, so ist davon auszugehen, dass die bewertete Wahrnehmung des Vereins die Bewertung der Stadt beeinflusst. Bei Betrachtung der Koordinatensysteme der Satellitenfans und der Outsider zeigt sich, dass genau dies festzustellen ist. Die positiven Attribute lagen in der Bewertung der Satellitenfans ausschließlich im oberen rechten Quadranten, sie trafen also aus ihrer Sicht auf den Verein und auf die Stadt zu. Die Gruppe der Outsider auf der anderen Seite schätzte die positiven Attribute als teils auf den Verein zutreffend ein (die Punkte liegen leicht oberhalb der x-Achse), jedoch treffen die Attribute aus ihrer Sicht erkennbar weniger auf die Stadt zu (die Punkte liegen linksseitig der y-Achse). Die hier durch die grafische Darstellung belegten Ergebnisse werden durch den Mittelwertvergleich in Tabelle 1 bestätigt: Tatsächlich bewerteten Satellitenfans nicht nur den Verein, sondern auch die Stadt Mönchengladbach hinsichtlich aller abgefragten Items signifikant besser als Outsider - ein deutlicher Hinweis darauf, dass ein positiver Imagetransfer vom Verein auf die Stadt Mönchengladbach stattfindet.

Ein überraschendes Ergebnis zeigt sich, wenn die Bewertungen der Eigenschaften anhand der Herkunftsregionen analysiert werden. Von den befragten Personen aus dem restlichen Deutschland wurde die Stadt Mönchengladbach bei allen positiven Eigenschaften am besten bewertet, bezüglich der Eigenschaften „,begeisternd“, „erfolgreich“, ,jung/dynamisch“ gaben die Befragten aus dem restlichen Nordrhein-Westfalen die zweitbeste Bewertung ab. Das Außenimage der Stadt Mönchengladbach scheint somit besser zu sein als der Eindruck der Fanregion-Bewohner. Dies zeigt sich auch in der Analyse der Bewertungen nach Fanclustern - hier war die Bewertung der Stadt durch die Satellitenfans besser als die der Heimfans und die Bewertung der Outsider leicht positiver als die Bewertung der regionalen Nicht-Fans. Die Tatsache, dass das Eigenimage der Stadt aus der Sicht der Bewohner der Fanregion negativer ausfällt als das Fremdimage der Stadt aus der Sicht der Befragten von außerhalb, ist ein weiterer Hinweis auf die hohe Bedeutung des Vereins für die Stadt. 
Abb. 8 Eigenschaften von Stadt und Verein im Vergleich Betrachtung nach ausgewählten Fanclustern. (Quelle: Eigene Darstellung auf der Basis der durchgeführten Erhebungen, $n=1.198$ )

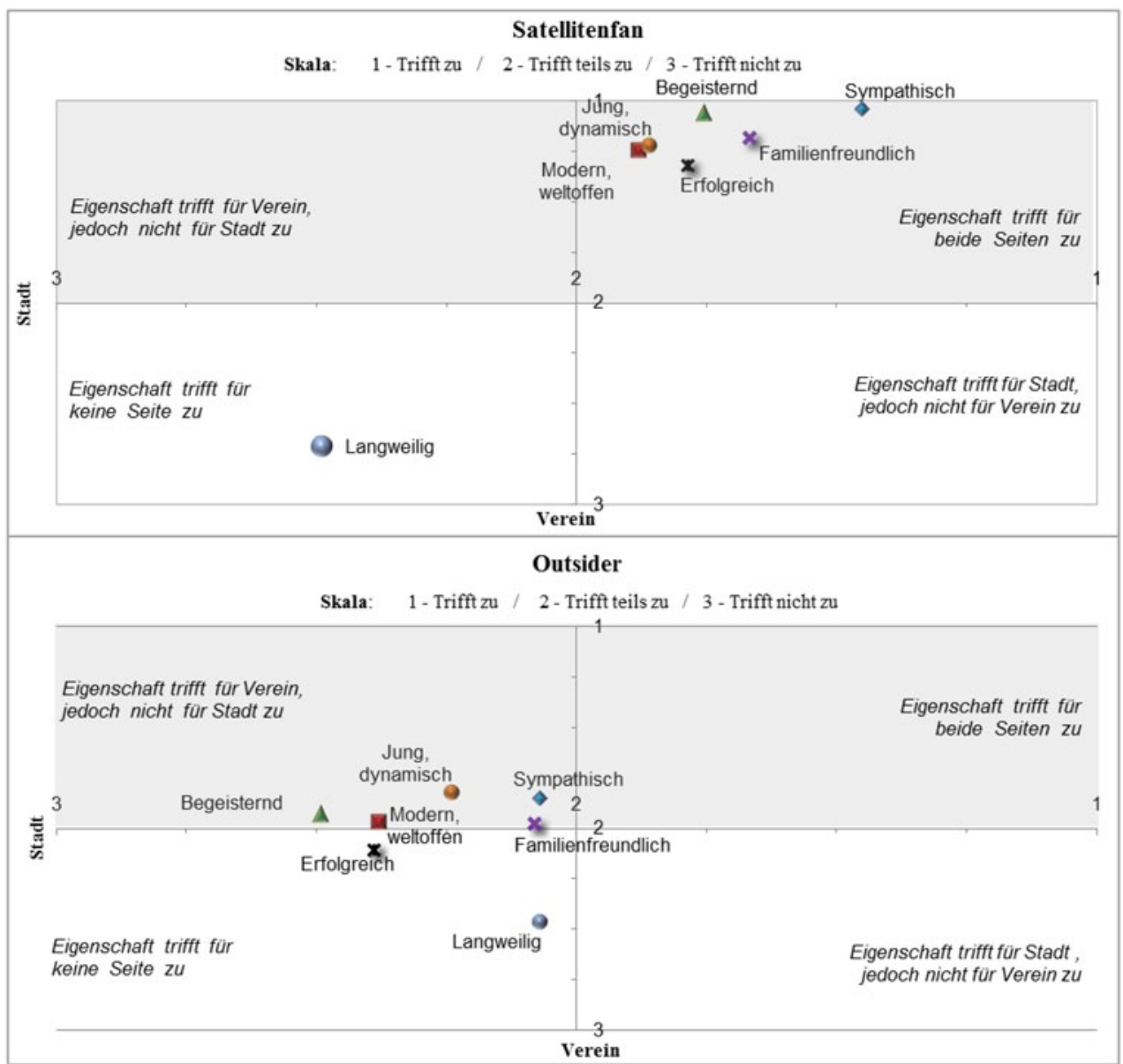

Tab. 1 Vergleich der Mittelwerte nach Fanclustern. (Quelle: Eigene Darstellung auf Basis der durchgeführten Erhebungen; die Anzahl der Antworten lag zwischen 146 und 322)

\begin{tabular}{lllrlll}
\hline Attribut & Mittelwert & Mittelwert differenz & \multicolumn{2}{l}{ Mittelwert } & Mittelwert differenz \\
\cline { 2 - 3 } \cline { 5 - 6 } & Satellitenfans & Outsider & & Heimfans & Regionale Nicht-Fans & $-0,426^{\mathrm{a}}$ \\
\hline Sympathisch & 1,45 & 2,07 & $0,621^{\mathrm{a}}$ & 1,73 & 2,15 & $-0,309^{\mathrm{a}}$ \\
Modern, weltoffen & 1,88 & 2,38 & $-0,499^{\mathrm{a}}$ & 2,07 & 2,38 & $-0,470^{\mathrm{a}}$ \\
Begeisternd & 1,76 & 2,49 & $-0,734^{\mathrm{a}}$ & 2,12 & 2,59 & $-0,301^{\mathrm{a}}$ \\
Familienfreundlich & 1,66 & 2,08 & $-0,410^{\mathrm{a}}$ & 1,84 & 2,14 & $-0,279^{\mathrm{a}}$ \\
Erfolgreich & 1,79 & 2,39 & $-0,608^{\mathrm{a}}$ & 2,16 & 2,44 & $-0,226^{\mathrm{a}}$ \\
Jung, dynamisch & 1,86 & 2,24 & $-0,385^{\mathrm{a}}$ & 2,12 & 2,35 & $0,152^{\mathrm{b}}$ \\
Langweilig & 2,49 & 2,07 & $0,424^{\mathrm{a}}$ & 2,22 & 2,07 & \\
\hline
\end{tabular}

Eigene Berechnungen

${ }^{a}$ Bei einem Signifikanzniveau von $<1 \%$ gesichert

${ }^{\mathrm{b}}$ Bei einem Signifikanzniveau von $<5 \%$ gesichert

\subsubsection{Standorteffekte}

Um direkte Attrahierungs- und Sogeffekte der Borussia auf die Standortentscheidungen anderer Unternehmen zu identifizieren, wurde analysiert, wie sich die ökonomische Aktivität im Umfeld des Borussia-Parks in den letzten Jahren entwickelt hat. Dabei war vielfältige Unternehmensaktivität festzustellen, die ohne den Bau des Stadions dort vermutlich nicht anzutreffen wäre. Der Borussia-Park befindet sich auf einem $535.000 \mathrm{~m}^{2}$ großen Areal. Es umfasst das Fußballstadion, das Trainingsgelände, das Verwaltungsgebäude, den Fan-Shop sowie eine Sportsbar und die medicoreha Borussia Mönchengladbach Sportrehabilitation GmbH. Zusätzlich können im Borussia-Park zahlreiche weitere Veranstaltungsräume wie die Logen, der Presseraum, der VIP-Bökelberg-Club und die Business Lounge für Events der verschiedensten Art angemietet werden. Über den üblichen Spielbetrieb hinaus finden jährlich rund 450 Ver- 
anstaltungen im Borussia-Park statt. ${ }^{11}$ Der Borussia-Park ist somit ein Anziehungspunkt, der über den Bundesligabetrieb hinaus aufgesucht werden kann.

Um direkte Sogeffekte für den Standort zu realisieren, ist aber nicht allein ein breites Angebot in der Nähe des Stadions bedeutsam, sondern auch die Einbindung der Sportstätten in ein integriertes und stimmiges Entwicklungskonzept von Städten und Regionen (Crompton 2004: 47). Die Stadt Mönchengladbach griff nach Abzug der britischen Truppen das Projekt „Nordpark“ auf und versuchte, die insgesamt 160 ha große militärische Konversionsfläche einer neuen Verwendung zuzuführen. Angestrebt wurde ein Gebiet für die zivile Nutzung, das die Bereiche „Sport, Wohnen, Arbeit und Freizeit" abdeckt. Begonnen hat die Neugestaltung des Nordparks 2004 mit dem Bau des Borussia-Parks. Sie setzte sich zeitnah fort durch die Errichtung des hochmodernen Warsteiner Hockeyparks, in dem 2006 die Hockeyweltmeisterschaft der Herren stattfand und der heute für musikalische Großveranstaltungen genutzt wird, sowie durch den Bau einer neuen gesamtstädtischen Finanzbehörde. Auch ein renommiertes Mönchengladbacher Textilunternehmen entschied sich dafür, seine Firmenzentrale in die Nähe des Borussia-Stadions zu verlegen (EWMG 2014). Das denkmalgeschützte Gebiet im Nordpark wurde zunehmend zum Anziehungspunkt für Dienstleistungsunternehmen, wie zum Beispiel Werbeagenturen. An dieser Stelle kann durchaus von einem Sogeffekt auf Unternehmensansiedlungen gesprochen werden; denn es erscheint fraglich, ob ein in der Peripherie gelegenes ehemaliges Militärgelände - ohne den Borussia-Park als Nukleus - Unternehmen dazu bewogen hätte, sich dort niederzulassen.

Was die Einbindung des Borussia-Parks in das Konzept des Nordparks anbelangt, kann von einem stimmigen und integrierten Entwicklungskonzept für das Stadion gesprochen werden. Trotzdem muss der Stadionstandort auch kritisch betrachtet werden. Im Gegensatz zur früheren Spielstätte, dem Bökelbergstadion, das sich in direkter Nähe zur Innenstadt befand, liegt der Borussia-Park in der Peripherie von Mönchengladbach. Er ist von der Innenstadt aus ohne die Nutzung von Verkehrsmitteln nur schwer erreichbar. Die meisten Fußballinteressierten reisen mit Auto, Bus und Bahn an, weil sie unmittelbar am Bahnhof die Busshuttles oder die zahlreichen Parkplätze am Stadion nutzen können. Spielbesucher, die mit dem Auto von außerhalb anreisen, werden aus verkehrstechnischen Gründen gezielt so über das Autobahnnetz geleitet, dass sie die Innenstadt umfahren und direkt am Stadion ankommen. Zugreisende Fußballfans von außerhalb werden sogar über den Bahnhof im benachbarten Rheydt zum Stadion geleitet. Durch diese Planung wird die Mönchengladbacher Innenstadt nur von

\footnotetext{
${ }^{11}$ Diese Information basiert auf Angaben des Vereins aus dem Jahr 2013.
}

wenigen Fans besucht. Die Innenstadt kann sich dementsprechend den Fußballgästen nicht präsentieren und kaum von ihnen profitieren.

$\mathrm{Zu}$ den indirekten Impulsen des Vereins aus der Sicht der Bevölkerung zählt die Verbesserung der weichen Standortfaktoren, also z. B. eine Steigerung der Lebensqualität der Bevölkerung durch ein verbessertes Heimatgefühl oder ein verbessertes Angebot für Freizeitaktivität. Die befragten Personen des verkleinerten Samples schätzten die Verbesserung des Angebots an Freizeitmöglichkeiten als zweitschwächsten Effekt mit einem Mittelwert von 2,99 ein (Skala von 1 - sehr stark bis 5 - überhaupt nicht). Dieser Effekt wird selbst von den Fans der Borussia (Mittelwert: 2,69) nur mittelmäßig wahrgenommen. Auffällig ist, dass insbesondere die Bewohner der Stadt und der Fanregion (Heimfans, regionale Nicht-Fans) die Verbesserung des Freizeitangebots kritischer beurteilen als die vom Fanstatus her vergleichbaren regionsexternen Befragten (Satellitenfans und Outsider). Offensichtlich wird gerade von den Bewohnern der Region das Engagement von Borussia Mönchengladbach bei der Förderung des regionalen Freizeitangebotes nur wenig wahrgenommen oder als nicht ausreichend angesehen, obwohl gerade die heimischen Befragten die größten Nutznießer dieses Angebots sein sollten.

In einer von der Industrie- und Handelskammer (IHK) Mittlerer Niederrhein in Auftrag gegebenen Unternehmensbefragung (Hamm/Wenke/Növer et al. 2013: 20) wird der Standortfaktor „Erholungs- und Freizeitmöglichkeiten“ als weniger wichtig eingestuft. Bedenkt man ferner, dass der Verein nur einen Bruchteil der regionalen Freizeitmöglichkeiten ausmacht, so ist dies ein klarer Hinweis darauf, dass die indirekten Standorteffekte eines Fußballvereins als äußerst gering einzustufen sind.

\subsubsection{Psychisches Einkommen}

Das „psychische Einkommen“ beschreibt den Nutzen, der für die Einwohner allein durch die Existenz des Vereins entsteht. Wie bereits ausgeführt, ist es insbesondere das Selbstwertgefühl und die Identifikation der Bevölkerung in der Stadt bzw. Fanregion mit ihrer Heimatstadt, in der sich das psychische Einkommen manifestiert. Auf der Basis der durchgeführten Befragung wird in Abbildung 9 dargestellt, inwieweit die Befragten glauben, dass die Borussia die Identifikation der Einwohner mit ihrer Stadt verbessert. Insgesamt schätzten im verkleinerten Sample 16,2\% der Befragten den Effekt des Vereins auf die Identifikation der Bevölkerung als sehr stark ein, nur 5,4\% sahen einen derartigen Effekt überhaupt nicht. Vergleicht man die Einschätzung der Fancluster, so zeigt sich, dass insbesondere Heimfans, aber auch Satellitenfans den Identifikationseffekt äußerst hoch einschätzten, deutlich höher als die Befragten, 
Abb. 9 Effekte des Vereins auf die Identifikation der Bevölkerung mit ihrer Heimatstadt. (Quelle: Eigene Darstellung auf der Basis der durchgeführten Erhebungen)

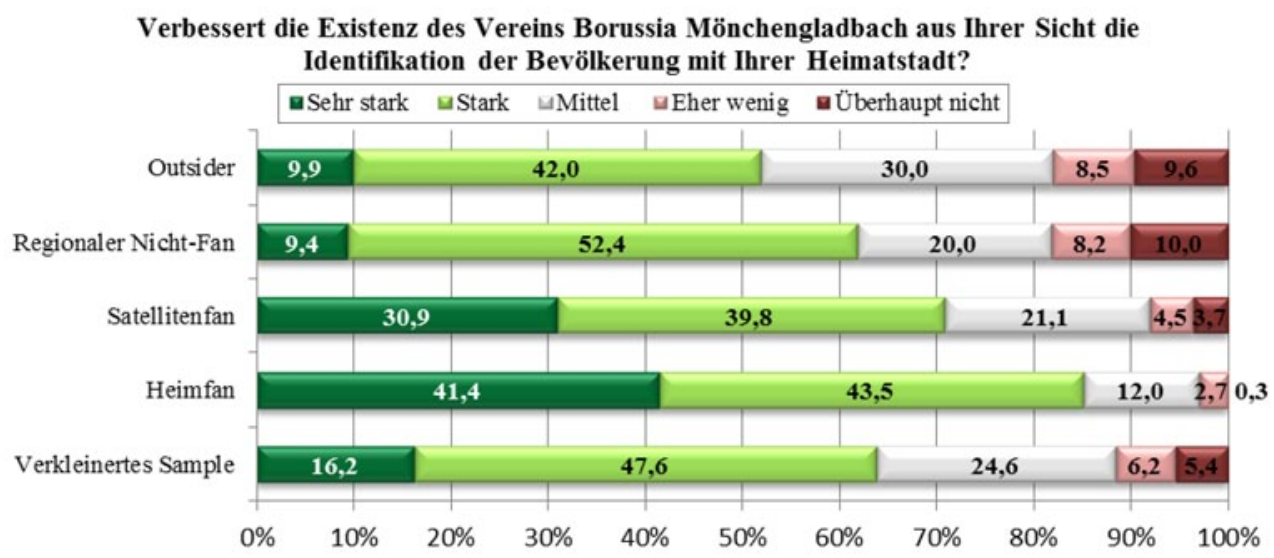

die keine Verbundenheit zum Verein angaben. Hier ähneln die Ergebnisse denen der Analyse von Imageeffekten des Vereins auf die Region.

Um den durch das psychische Einkommen entstehenden Nutzen für die Bevölkerung in einer ersten Annäherung ungefähr abschätzen zu können, wurden die Befragten zusätzlich nach ihrer Spendenbereitschaft bei einem drohenden finanziellen „Aus“ der Borussia gefragt. Erwartungsgemäß waren die Vereinsfans deutlich eher bereit, hohe Summen für den Vereinserhalt zu spenden, das heißt, bei ihnen war die psychologische Bedeutung des VfL deutlich höher als bei Sympathisanten und Nicht-Fans. Ein durchschnittlicher Heimfan war bereit, etwa $110 € \mathrm{zu}$ spenden, ein durchschnittlicher Satellitenfan zeigte eine Spendenbereitschaft von knapp $98 €$. Dennoch ist anzumerken, dass auch ca. 20\% derjenigen Befragten, die angaben, keinerlei Verbundenheit zum Verein zu haben, zu einer Spende bereit wären - das psychische Einkommen dürfte demnach auch in dieser Bevölkerungsgruppe ,fühlbar' sein. So lag die durchschnittliche Spendenbereitschaft der Bewohner der Fanregion, die sich als Sympathisanten des Vereins einstuften, bei mehr als $30 €$, bei regionalen Nicht-Fans immerhin noch bei rund $7 €$. Es zeigt sich, dass die Spendenbereitschaft mit steigender Intensität der Fanbeziehung wächst - es überrascht kaum, dass mit zunehmender Vereinsbegeisterung auch das psychische Einkommen steigt.

Ausgehend von der Annahme, dass die Spendenbereitschaft ein Indikator für das „psychische Einkommen“ ist, wurde versucht, das gesamte psychische Einkommen zu quantifizieren, welches für die Bevölkerung der Stadt Mönchengladbach bzw. der Fanregion durch die Existenz des Vereins entsteht. Dazu wurde die durchschnittliche (regionale) Spendenbereitschaft des verkleinerten Samples auf die (regionale) Gesamtbevölkerung über 16 Jahre hochgerechnet $^{12}$ : Für die Stadt Mönchengladbach ergab die

\footnotetext{
${ }^{12}$ Datenbasis Regionaldatenbank des Statistischen Bundesamtes:
} Bevölkerungszahl über 15 Jahre in den Kreisen der Fanregion am
Hochrechnung eine Spendenbereitschaft der Bevölkerung von fast 10 Mio. $€$. Für die Fanregion wurde sogar eine Spendenbereitschaft von etwa 38,5 Mio. $€$ ermittelt.

\section{Zusammenfassung und Schlussfolgerung}

Die Zielsetzung des vorliegenden Beitrags bestand darin, anhand des ausgewählten Fallbeispiels ein möglichst umfassendes Bild der regionalwirtschaftlichen Effekte von Fußballvereinen zu zeichnen. Die Analyse hat zunächst gezeigt, dass die regionalen Wertschöpfungs-, Einkommensund Beschäftigungseffekte von Borussia Mönchengladbach nicht unerheblich sind. Für den Niederrhein liegen die vereinsbedingten Wertschöpfungseffekte bei fast 100 Mio. $€$; 1.075 Arbeitskräfte werden benötigt, um diese Wertschöpfung zu generieren. Da andere Industrie- und Dienstleistungsunternehmen in der Regel keine Ausgaben und dadurch induzierte Effekte durch hohe Besucherzahlen generieren, übersteigen bereits diese nachfrageseitigen Wirkungen die regionalwirtschaftlichen Effekte eines ,normalen Unternehmens'.

Die vorangegangene Analyse macht aber auch deutlich, dass der Verein Borussia VfL 1900 Mönchengladbach für seine Standortkommune weitere, intangible Effekte hat. Da diese Effekte monetär nicht quantifizierbar sind, muss offen bleiben, ob ihre Höhe (bzw. Wichtigkeit) die der nachfrageseitigen Effekte übersteigt. Zumindest die Befragungsteilnehmer stuften die Bedeutung der im Beitrag diskutierten intangiblen Effekte höher ein als die (monetären) Wirkungen auf die Einzelhandelsumsätze.

31.12.2011: Mönchengladbach - 222.518 Einwohner; Rhein-Kreis Neuss - 381.830 Einwohner; Kreis Viersen - 259.051 Einwohner; Kreis Heinsberg - 218.636 Einwohner (eigene Berechnung nach Angaben von Information und Technik Nordrhein-Westfalen 2016). 
Im Einzelnen kann festgehalten werden:

- Wahrnehmung der Stadt: Der Verein trägt dazu bei, die Stadt Mönchengladbach international, insbesondere aber national bekannt zu machen.

\section{- Image der Stadt:}

- Borussia Mönchengladbach kann als das wichtigste Wahrzeichen und als bedeutender Imageträger der Stadt angesehen werden.

- Bei der Abfrage verschiedener Image-Attribute schneidet der Verein deutlich positiver ab als die Stadt.

- Eine Kombination von grafischer Veranschaulichung und Mittelwertvergleichen zeigt, dass Fans des Vereins nicht nur den Verein, sondern auch die Stadt Mönchengladbach bei den benutzten ImageItems signifikant besser beurteilen als Nicht-Fans. Dies kann als ein deutlicher Hinweis darauf gewertet werden, dass ein positiver Imagetransfer vom Verein auf die Stadt Mönchengladbach erfolgt.

Der Verein trägt somit dazu bei, das Image der Stadt Mönchengladbach - einen für die Standortentscheidungen von Unternehmen und für die Wohnortentscheidungen von Haushalten entscheidungsrelevanten Faktor - zu verbessern.

- Standorteffekte: Bei den angestellten qualitativen Überlegungen wurde Unternehmensaktivität festgestellt, die ohne die Existenz von Stadion und Verein dort vermutlich nicht anzutreffen wäre. Diese Aktivität ist zum überwiegenden Teil den direkten Sogwirkungen zuzurechnen. Theoretisch ebenfalls denkbare indirekte Sogwirkungen sind hingegen nicht zu identifizieren.

- Psychisches Einkommen: Auf die Existenz eines psychischen Einkommens deuten zum Ersten die Befragungsergebnisse hin: Die Befragten waren mehrheitlich davon überzeugt, dass es Identifikationswirkungen gibt, die die Grundlage eines derartigen Einkommenseffektes sind. Aufbauend auf der Annahme, dass Spendenbereitschaft als Indikator für dieses „psychische Einkommen“ angesehen werden kann, ist mithilfe der erhobenen Daten zum Zweiten auch eine - sicherlich methodisch nicht unbedenkliche - erste Abschätzung der Höhe des psychischen Einkommens möglich. Nach den angestellten Hochrechnungen würde sich dies für die Stadt Mönchengladbach auf fast 10 Mio. $€$, für die Fanregion sogar auf mehr als 38,5 Mio. $€$ belaufen.

Durch die beachtlichen monetären regionalwirtschaftlichen Effekte im nachfrageseitigen Bereich werden die angebotsseitigen Wirkungen der Borussia auf die Stadt Mönchengladbach, insbesondere hinsichtlich Bekanntheit, Image und Identifikation der Bevölkerung mit ihrer Stadt, übersehen oder unterschätzt. Als überregional bekanntes
Wahrzeichen und als wichtiger Imageträger nimmt der Verein Borussia VfL 1900 Mönchengladbach eine bedeutende Funktion für die Stadt Mönchengladbach wahr und stellt ein zentrales Potenzial für das Stadtmarketing dar. Als altindustriell geprägte Stadt musste Mönchengladbach im Strukturwandel einen starken Rückgang seiner traditionellen Industrien hinnehmen und muss bis heute den damit verbundenen strukturellen Anpassungsbedarf bewältigen. Im Standortwettbewerb mit anderen nordrhein-westfälischen Städten wie z. B. Köln, Düsseldorf oder Aachen, die über ein höheres ökonomisches Potenzial verfügen, sind positive Image- und Bekanntheitsgradeffekte für die Stadt Mönchengladbach daher von großer Wichtigkeit. Der Verein ist durch seine regionalwirtschaftlichen Effekte eine wichtige Stütze in diesem Wettbewerb mit anderen Städten.

\section{Einhaltung ethischer Richtlinien}

Interessenkonflikt Es besteht kein Interessenkonflikt.

\section{Literatur}

Ahlert, G. (2013): Die wirtschaftliche Bedeutung des Sports in Deutschland. Abschlussbericht zum Forschungsprojekt „Satellitenkonto Sport 2008“ für das Bundesinstitut für Sportwissenschaft (BISp). http://www.gws-os.com/discussionpapers/ gws_researchreport13-2.pdf (20.07.2015).

Crompton, J. L. (1995): Economic Impact Analysis of Sports Facilities and Events: Eleven Sources of Misapplication. In: Journal of Sport Management 9, 1, 14-35.

Crompton, J. L. (2004): Beyond Economic Impact: An Alternative Rationale for the Public Subsidy of Major League Sports Facilities. In: Journal of Sport Management 18, 1, 40-58.

Dietl, M.; Pauli, M. (1999): Wirtschaftliche Auswirkungen öffentlich finanzierter Stadionprojekte. Paderborn. = Arbeitspapiere der Universität Paderborn 61.

Diller, C. (1991): Weiche Standortfaktoren. Zur Entwicklung eines kommunalen Handlungsfeldes. Das Beispiel Nürnberg. Berlin. $=$ Arbeitshefte des Instituts für Stadt- und Regionalplanung der Technischen Universität Berlin 43.

Essig, C.; Soulas de Russel, D.; Semanakova, M. (2003): Das Image von Produkten, Marken und Unternehmen. Sternenfels.

EWMG - Entwicklungsgesellschaft der Stadt Mönchengladbach mbH (2014): Projekt Nordpark. Visionen und Potentiale. http://ewmg. de/projekt-nordpark.html (13.01.2014).

Grabow, B. (1994): „Weiche“ Standortfaktoren. In: Dieckmann, J.; König, E. M. (Hrsg.): Kommunale Wirtschaftsförderung - Handbuch der Standortsicherung und -entwicklung in Stadt, Gemeinde und Kreis. Stuttgart, 147-163.

Hamm, R. (1998): Regionalwirtschaftliche Effekte eines Fußballbundesligisten - Theoretische Überlegungen und einige empirische Ergebnisse. In: Raumforschung und Raumordnung $56,1,43-48$.

Hamm, R. (1999): Regionalwirtschaftliche Effekte von Events und Probleme bei ihrer Quantifizierung - Überlegungen am Beispiel eines Fußball-Bundesligavereins. In: Tourismus Journal 3, 3, 303-324. 
Hamm, R.; Jäger, A.; Fischer, C. (2014): Regionalwirtschaftliche Effekte eines Fußball-Bundesliga-Vereins - Dargestellt am Beispiel des Borussia VfL 1900 Mönchengladbach. Mönchengladbach. $=$ Mönchengladbacher Schriften zur wirtschaftswissenschaftlichen Praxis 27.

Hamm, R.; Janßen-Timmen, R.; Moos, W. (2006): Die wirtschaftliche Bedeutung des VfL Borussia Mönchengladbach für den Niederrhein. Gutachten im Auftrag von Borussia Mönchengladbach. Mönchengladbach (unveröffentlichtes Manuskript).

Hamm, R.; Vetter, R. (2004): Kommunalwirtschaftliche Analysen als Instrument der Politikberatung - Eine Darstellung am Beispiel der Stadt Korschenbroich. Aachen. = Mönchengladbacher Schriften zur wirtschaftswissenschaftlichen Praxis 15.

Hamm, R.; Wenke, M.; Növer, R.; Werkle, G. (2013): Wirtschaftliche Strukturen und Entwicklungen im IHK-Bezirk Mittlerer Niederrhein. Krefeld. $=$ IHK-Schriftenreihe 135 .

Hassenpflug, F. (2012): Wirtschaftsfaktor VfL Osnabrück. Studie über ökonomische Auswirkungen des Fußballvereins in Stadt und Region (Kurzfassung). http://www.vfl.de/fileadmin/vfl/ Redakteur/PDF-Dokumente/Wirtschaftsfaktor_VFL_Web.pdf (24.09.2013).

Information und Technik Nordrhein-Westfalen (2016): Kommunales Bildungsmonitoring: Tab. A1.2, Bevölkerung nach Altersgruppen und Geschlecht (Basis VZ 87). https://www.landesdatenbank. nrw.de/ldbnrw/online/logon (16.02.2016).

Luh, A. (2002): Wir sind die Ruhrpott-Kanaken: Fußball und Identität im Ruhrgebiet 1920-2000. In: Krüger, A.; Buss, W. (Hrsg.): Transformationen: Kontinuitäten und Veränderungen in der Sportgeschichte II. Göttingen, 203-211. = Schriftenreihe des Niedersächsischen Instituts für Sportgeschichte Hoya e. V. 17.
Misoch, S. (2015): Qualitative Interviews. Berlin, München, Boston. Preuß, H.; Könecke, T.; Schütte, N. (2010): „Ökonomische Auswirkungen des 1. FC Kaiserslautern für Kaiserslautern und Rheinland-Pfalz“. Ergebniszusammenfassung der Studie. http://www.sport.uni-mainz.de/Preuss/Download\%20public/ projekte/20100510_FCK_Studie_Ergebnisse.pdf (24.09.2013).

Schmidt, S. L.; Bünning, F. (2012): Die Stadt und ihr Profifußball: Eine ganz normale Beziehung. https:/www.ebs.edu/fileadmin/ redakteur/funkt.dept.sol/ISBS/ISBS_Issue 5 Die_Stadt_und ihr Profifussball.pdf (24.09.2013). $=$ ISBS Research Series 5 .

Schwark, J. (2004): Regionale Wertschöpfung von Sport(groß) veranstaltungen. In: Krüger, A.; Dreyer, A. (Hrsg.): Sportmanagement: Eine themenbezogene Einführung. München, Wien, 193-210.

ServiceValue GmbH (2013): Familienfreundliche Unternehmen. http:// www.servicevalue.de/wettbewerbe/branchenuebergreifend/ familienfreundliche-unternehmen/ (03.01.2014).

Siegfried, J.; Zimbalist, A. (2000): The Economics of Sports Facilities and Their Communities. In: Journal of Economic Perspectives 14, 3, 95-114.

Statistisches Bundesamt (2010): Volkswirtschaftliche Gesamtrechnungen, Input-Output-Rechnung. https://www.destatis. de/DE/Publikationen/Thematisch/VolkswirtschaftlicheGesam trechnungen/InputOutputRechnung/VGRInputOutputRechn ung2180200079004.pdf?_blob=publicationFile (10.01.2014)

Vöpel, H.; Steinhardt, M. (2008): Wirtschaftsfaktor Fußball - Globale Entwicklungen und die regionalwirtschaftlichen Potenziale des HSV. Studie im Auftrag der HSH Nordbank AG. www.hwwi. org/fileadmin/hwwi/Publikationen/Partnerpublikationen/HSH/ Fussballstudie_14_B.pdf(24.09.2013). 